\title{
Sample preparation for the analysis of membrane proteomes by mass spectrometry
}

\author{
Xianchun Wang, Songping Liang \\ College of Life Sciences, Hunan Normal University, Changsha 410081, China \\ $\triangle$ Correspondence: liangsp@hunnu.edu.cn \\ Received July 3, 2012 Accepted July 18, 2012
}

\begin{abstract}
The low abundance and highly hydrophobic nature of most membrane proteins make their analysis more difficult than that for common soluble proteins. Successful membrane protein identification is largely dependent on the sample preparation including the enrichment and dissolution of the membrane proteins. A series of conventional and newly developed methods has been applied to the enrichment of low-abundance membrane proteins at membrane and/or protein levels and to the dissolution of hydrophobic membrane proteins. However, all the existing methods have inherent advantages and limitations. Up to now, there has been no unique method that can universally be employed to solve all the problems and more efforts are needed in improving sample preparation for the analysis of membrane proteomes.
\end{abstract}

KEYWORDS sample preparation, membrane proteome, enrichment, extraction, digestion, mass spectrometry

\section{INTRODUCTION}

Membranes are critical components of cellular structure and function, involving the partitioning of organelles, protecting the integrity of the genome and proteome, and providing defense against foreign molecules and external conditions that may damage or destroy the cell (Blonder et al., 2002). The functions of cells are closely related to the proteins present in the membranes because the membrane proteins are major components of the membranes and carry out many essential biological membrane functions. Among the membrane proteins, the plasma membrane (PM) proteins are of particular importance because they play a crucial role in the fundamental biological process of the cell, including the ex- change of the material and energy between the cell and its environment, cell-cell interactions, and signal transport. A better knowledge of the membrane proteins would greatly help to understand the highly diverse structure and functions of the cell. However, although there have been great advances in protein chemistry and proteomics in recent years, the analysis of membrane proteins still presents an analytical challenge because most membrane proteins are not only present in low-abundance, but also they are not readily soluble in pure aqueous buffers due to their hydrophobicity and tendency for aggregation. These properties of membrane proteins undoubtedly lead to the low number and coverage of proteins identified. Therefore, efficient membrane proteome analysis, e.g., by mass spectrometry, is largely dependent on appropriate sample preparation, which involves membrane enrichment and the extraction, separation, proteolysis of the proteins and recovery of the digests, etc.

\section{MEMBRANE ENRICHMENT}

For the analysis of membrane proteins, particularly those with low abundance, enrichment of membranes is the crucial first step. The most used methods are traditional differential centrifugation and density gradient centrifugation. Using these methods, the purity of PMs can be increased about 10-fold (Cao et al., 2006). However, due to the overlapping densities, it is usually difficult for a particular subcellular membrane to be completely purified by density gradient centrifugation. PM fractions prepared by this method, for example, are often heavily contaminated with mitochondrial and other cellular inner membranes. In addition, this method is not well suited for a small amount of sample. In practice, these traditional methods are often employed in combination with other enrichment approaches to obtain membrane samples with even higher purity.

Partitioning of membranes in aqueous-polymer two-phase 
systems (two-phase partition method) is a method for affinity purifying PMs that was widely used in 1980's because it was believed to be able to separate mitochondria and other cellular inner membranes from PMs on the basis of their different surface properties. In an aqueous two-phase partition system composed of polyethylene glycol (PEG) and dextran, PMs tend to enter the PEG-rich upper phase, whereas mitochondria and other subcellular organelles tend to enter the dextran-rich bottom phase (Walter et al., 1985; Morré et al., 1989). Using the two-phase partition method, in combination with differential centrifugation and density gradient centrifugation, the concentration of PMs, as determined by the $\mathrm{Na}^{+} / \mathrm{K}^{+}$ATPase marker was about 25 times higher than that seen in the total homogenate and 2.5 times higher than in the crude PM fraction prepared using only differential centrifugation and density gradient centrifugation (Cao et al., 2006). When the two-phase partition method was used in combination with only differential centrifugation, the relative concentration of PMs was about 15 times higher than that in the total homogenate (Xiong et al., 2009). To further improve the enrichment and purification, a secondary partitioning step, employing a PM-specific affinity ligand such as wheat germ agglutinin conjugated to one of the polymers, generally dextran, can be used. During the process, the contaminating membranes left over from the first partitioning step partition into the PEG phase, while the affinity ligand causes the target membrane to partition into the dextran phase (Schindler et al., 2006). Since the two-phase partition method is sensitive to the polymer concentration, salt, temperature, affinity ligand, etc., application of the method requires carefully controlled conditions and optimization for specific tissue sources (Schindler and Nothwang, 2006; Speers and Wu, 2007).

For preparation of PM fractions of high purity, integration of density gradient centrifugation and immunoaffinity using antibodies directed against special membrane proteins is another choice. The antibodies are bound to immunoaffinity magnetic beads in order to select for PMs. After the PM-enriched fraction from the sucrose density gradient is incubated with the magnetic beads suspended in buffer, the bead complexes are collected by placing the reaction tube in a magnet stand. Using this strategy, the PMs in the fraction could be enriched 3-fold in comparison with the density gradient centrifugation method (Zhang et al., 2007). In addition, many other methods for membrane enrichment, such as a biotinylation method based on biotin-avidin system or a "colloidal silica coating" method that involves using cationic colloidal silica to coat the intact cells, can also be used alone or in combination with other selected methods (Li et al., 2009)

Use of high salt and high pH solutions to wash the membrane debris prepared with above methods can further improve the purity of membrane preparation because they reduce non-covalent protein-protein interactions, thus lowering the amount of non-membrane proteins that are adsorbed to PMs (Wu et al., 2003). For example, Marmagne et al. (2004) used chloroform/methanol, $\mathrm{NaOH}$ and nonionic detergents to extract and treat the membrane fraction purified from $A$. thaliana cells by differential centrifugation and aqueous twophase partitioning. Bartee et al. (2006) employed sodium carbonate $(\mathrm{pH} 11.5)$ and ammonium bicarbonate $(\mathrm{pH} 8.5)$ to sequentially rinse the membranes purified from Hela-Tet Off cells using discontinuous sucrose gradient ultracentrifugation to remove adsorbed undesirable proteins. Through these measures, the effectiveness of identification of membrane proteins was enhanced and the identified membrane proteins were $50 \%-60 \%$ of the total proteins identified. It should be noted that such washing cannot remove the membrane-associated proteins completely and repeated washing would lead to loss of certain peripheral membrane proteins.

It is worth mentioning that, apart from membrane enrichment, separation or fractionation at the protein and/or peptide level could also increase the rate of successful identification of low abundance proteins. Relatively, the separation at protein level is less frequently used, because the conventional 2D-PAGE is not compatible with the membrane proteomics and multidimentional liquid chromatorapgy is not suitable for the fractionation of proteins in limited amounts. The most widely used method at peptide level is multidimentional protein identification technology (Mudpit). For example, by using MudPIT, Washburn et al. (2001) identified a total of 1484 proteins from the Saccharomyces cerevisiae strain BJ5460, including a large number of proteins rarely seen in proteome analysis, including low-abundance proteins like transcription factors and protein kinases. Reinders et al. (2006) applied MudPIT to the yeast mitochondrial proteome and identified 491 proteins including many low-abundant membrane proteins and hydrophobic pepetides.

\section{ON-MEMBRANE DIGESTION}

On-membrane digestion means that the membrane proteins are directly digested on the membrane debris without being extracted from the membranes in advance. On-membrane digestion can reduce the number of non-membrane proteins and increase the purity of real membrane proteins, thus improving identification of the set of proteins. Although the membrane proteins in the membrane fraction obtained with the methods mentioned above are enriched compared with those in whole-cell lysate, the identification efficiency of membrane proteins, in particular integral membrane proteins, is often still far from satisfactory. A certain percentage of the identified proteins are usually contaminating proteins and membrane-associated proteins such as cytoskeletal proteins. The real membrane proteins, in particular low-abundant integral membrane proteins, including regulatory proteins and ion channel proteins, are mostly outside the scope of standard proteomic techniques. One of the main reasons for this is that some proteins absorbed on the membrane are difficult to remove by repeated washing and dominate the relatively 
small amounts of membrane proteins. In order to increase the representation of membrane proteins in the membrane fraction, repeated washing is often used in combination with on-membrane digestion. On-membrane digestion with proteases is performed in the presence or the absence of additives in order to remove undesirable proteins and to cleave the peripheral membrane proteins as well as exposed domains of integral membrane proteins from the membranes. Blonder et al. (2002) developed a membrane sample preparation method combining carbonate extraction, surfactantfree organic solvent-assisted solubilization and on-membrane proteolysis in the presence of $60 \%$ methanol. Nielsen et al. (2005) depleted non-membrane molecules from the entire tissue homogenate using high-salt, carbonate, and urea washes, followed by treatment of the membranes with sublytic concentrations of digitonin and protein digested on-membrane by endoproteinase Lys-C. Fischer et al. (2006) described a procedure for membrane and integral membrane protein enrichment involving both washing with high salt and high $\mathrm{pH}$ solutions and on-membrane digestion in their attempts toward elucidating the complete membrane proteome. Results from the foregoing studies demonstrated that the membrane sample preparation methods can indeed be improved, allowing enrichment and identification of membrane proteins, including integral membrane proteins. However, these techniques involved multiple rinsing and/or pre-digestion steps, which unavoidably lead to the loss of some membrane proteins and peptides. Furthermore, after extraction with organic solvent and on-membrane digestion, the remaining membrane fragments were removed and discarded, leading to the loss of another part of membrane proteins.

Zhou et al. (2008) developed a two phase on-membrane digestion method and applied it to the analysis of the rat liver membrane proteome. The two phase system was constituted

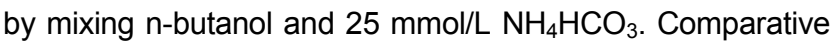
experiments indicated that the proteins on membranes could be digested in the two phase system more efficiently than in either $60 \%$ methanol or $25 \mathrm{mmol} / \mathrm{L} \mathrm{NH}_{4} \mathrm{HCO}_{3}$ solutions under the same conditions, thereby improving the identification of the membrane proteins. Using the established two phase system and CapLC-MS/MS to analyze rat liver membrane proteome, a total of 411 membrane proteins were identified, more than $80 \%$ of which were transmembrane proteins with 1-12 mapped transmembrane domains (TMDs). Due to its extraction and dissolution actions, the developed two phase on-membrane digestion system could efficiently improve the digestion and removal of adsorbed non-membrane proteins, and markedly increase the number and coverage of identified membrane proteins, particularly the transmembrane proteins. Furthermore, use the method to identify a complementary protein set from all fractions of the two phase system resulted in a higher coverage of the membrane proteome.

On-membrane digestion is often carried out in the presence of additives such as methanol, formic acid, urea and detergents. During the digestion process, the additives destabilize the membranes and enhance the solubility of the membrane proteins, thus improving the digestion of the proteins. Obviously, the additives added must be compatible with protease activity. In most cases, it is difficult for an additive both to effectively enhance the solubility of membrane proteins and to be compatible with protease activity. However, sodium deoxycholate (SDC) was found to be compatible with trypsin activity, with enzyme activity not being significantly influences by concentrations up to $2 \%$ (Zhou et al., 2006; Lin et al., 2008). Zhou et al. (2006) evaluated the use of SDC in place of $0.1 \%$ SDS for on-membrane digestion of rat hippocampal plasma membrane fraction as assessed by MALDI-TOF MS and CapLC-MS/MS. The use of SDC resulted in more total membrane protein identifications (71 vs. 31$)$ and more integral membrane proteins (IMPs) (50 vs. 22) by CapLC-MS/MS. Furthermore, because SDC precipitates at low pH, it can be removed from the digests by centrifugation following acidification prior to sample analysis, preventing any potential ion suppression (Zhou et al., 2006; Lin et al., 2010). In addition, Zhou et al. (2006) also demonstrated that during in-gel digestion the addition of $0.1 \%$ SDC in the buffer could remarkably enhance the coverage of tryptic peptides and the number of hydrophobic membrane proteins identified.

\section{MEMBRANE PROTEIN EXTRACTION}

Relatively, on-membrane digestion strategy has been less frequently employed in membrane proteomics. The membrane proteins are often extracted from the membranes before being digested. To enhance the solubility of membrane proteins and facilitate the extraction of these proteins, researchers have commonly used some additives such as organic solvents and detergents in their extraction buffers. In regard to the solubilization of membrane proteins, Masuda et al. (2008) evaluated the solubilization ability of 27 additives, including commonly used surfactants, chaotropes and organic solvents on the membrane-enriched fraction of $E$. coli lysate, and found that the anionic detergent SDS had the strongest ability to solubilize the proteins in the membrane fraction. Reynolds et al. (1970) also demonstrated that SDS is extremely effective for solubilizing and denaturing proteins and can dissolve a wide range of proteins, including misfolded and precipitated proteins. Unfortunately, although these additives can improve the solubilization and extraction of the membrane proteins to different degrees, most of them reduce the enzyme activity, interfere with high-performance liquid chromatography (HPLC) separation, and affect the mass spectrometric analysis. For example, SDS can be quite problematic in membrane proteomics because a slightly higher concentration of SDS can severely reduce the activity of proteolytic enzymes as well as interfering with subsequent analyses. To overcome this problem, some researchers have attempted to replace SDS with additives such as SDC, urea or methanol which interfere 
less with enzyme activity and/or mass spectrometry. However, these alternative additives were found to have a major drawback in that their ability to disrupt the membranes and extract highly hydrophobic proteins such as those with multiple transmembrane domains is weaker than that of SDS (Masuda T., 2008). In view of these facts, much effort has been made to remove the SDS from the SDS-solubilized protein samples (i.e. sample cleanup).

\section{GEL-BASED SAMPLE CLEANUP}

For sample cleanup, many conventional and newly developed methods have been employed, including dialysis, precipitation with organic solvent or organic acid, as well as column-, gel- and spin filter-based approaches (such as ion exchange, hydrophilic interaction chromatography, gel filtration, tube gel digestion and spin filter microcentrifugation). Although dialysis and column-based approaches have achieved some improvements in the cleanup of protein samples, these methods may not be suitable for high-throughput proteomic analysis because the large volume and complex operations introduced by these methods could result in significant sample and time losses during SDS removal and/or protein concentration (Andersen et al., 1993). Although the spin filter-based SDS removal methods, such as filter-aided sample preparation strategy (Manza et al., 2005; Wiśniewski et al., 2009), have seen some applications to the sample preparation for shotgun proteomics, these methods have their inherent limitations: (1) the peptide/protein recovery is low due to the strong binding of proteins and peptides to the spin filters and therefore it is not suitable for the analysis of small amounts of protein samples $(<50 \mu \mathrm{g})$; (2) there are difficulties in the removal of detergents from the highly hydrophobic proteins due to their tight binding. The approach is useful in some applications, but is not necessarily as "universal" as implied (Liebler et al., 2009; Wiśniewski et al., 2009). Recently, a gel-based method for the cleanup of SDS-solubilized membrane protein samples, named "tube-gel digestion," has been developed and applied (Lu et al., 2005). In this protocol, SDS-containing protein lysate was mixed with acrylamide solution, and the proteins were incorporated into the polyacrylamide gel when the gel solution polymerized. Detergents were removed by washing prior to protein digestion so as not to interfere with the subsequent analyses. However, this method unavoidably leads to significant protein loss, because there is usually a part of the protein solution excluded from the gel during gel polymerization. In addition, proteins incorporated into gel in this method have a higher risk of being chemically modified by reagents such as acrylamide (Hudgin and Ashwell, 1974; Andersen and Heron, 1993). Such limitations of the protocol will reduce the efficiency and confidence of protein identification and quantification.

Zhou et al. (2010a, b) have developed a dried gel absorp- tion-based sample preparation method for the cleanup and analysis of membrane proteomes. In this new method, membrane proteins solubilized in a starting buffer containing high concentration of SDS were directly entrapped and immobilized into the gel matrix when the membrane protein solution was absorbed by the vacuum-dried polyacrylamide gel. After the detergent and other salts were removed by washing, the proteins were subjected to in-gel digestion and the tryptic peptides were extracted and analyzed by CapLC-MS/MS. The results showed that this method not only avoided protein loss and the adverse protein modifications during gel-embedment, but also improved the subsequent in-gel digestion and the recovery of tryptic peptides, particularly the hydrophobic peptides, thereby facilitating the identification of membrane proteins, especially the integral membrane proteins. Compared with the above-mentioned tube-gel digestion method, the newly developed method increased the numbers of identified membrane proteins and integral membrane proteins by $25 \%$ and $30 \%$, respectively. By using the gel as a "proteome reactor," interference removal, buffer exchange, protein digestion and digest recovery were all readily achieved.

Somewhat earlier, Liu et al. (2008) developed a three-layer sandwich gel electrophoresis that could simultaneously remove salts and concentrate the sample. However, like other gel-based methods, this method could not separate protein mixture and needed additional fractionation for efficient protein identification. More recently, Liu et al. (2012) described a special gradient gel electrophoresis (GGE) system that can both remove SDS from the SDS-solubilized protein samples and fractionate proteins. The GGE system is composed of an agarose loading layer, six polyacrylamide fractionation layers with different concentrations and a high-concentration polyacrylamide sealing layer. The advantages of the GGE system are that it can not only electrophoretically remove SDS efficiently so that the protein loss resulting from the repeated gel washing after electrophoresis is avoided, but also can reduce the complexity of the sample, prevent the precipitation of proteins after loading and avoiding the loss of low molecular weight proteins during the electrophoresis. Using the GGE system, about $85 \%$ of SDS in the sample and gel is electrophoretically removed and the proteins are fractionated. Compared with the other representative gel-based sample cleanup methods reported in literature, GGE-based strategy significantly improves the efficiency of identification of proteins in terms of the number and coverage of the identified proteins.

\section{ENTIRELY SOLUTION-BASED SAMPLE PREPARATION}

Although the gel-based method is effective in the membrane protein sample cleanup, the method has its inherent limitations, including that it is not suitable for treating the large-volume sample and that the proteins cleaned up by this 
method are generally needed to be digested in the gel (in-gel digestion), which has disadvantages such as low accessibility of proteases to the gel-entrapped proteins, low cleavage yields of some proteins and difficulties in extraction of large and/or hydrophobic peptides from the gel matrix (LuqueGarcia et al., 2006). Compared with the in-gel digestion, in-solution digestion can provide a liquid environment more suitable for subsequent digestion, which avoids some disadvantages of in-gel digestion. For in-solution digestion of hydrophobic membrane proteins and other difficultly dissolved proteins, it is crucial to clean up the samples with a solution-based method after these proteins have been extracted with strong detergents such as SDS.

Of the solution-based methods that can be used to clean up protein samples, protein precipitation with organic solvents, especially with cold acetone, has attracted special attention and found some applications (Puchades et al., 1999; Botelho et al., 2010). The method operates simply and can reduce the concentration of small-molecular-weight interfering substances in protein samples, thus reducing the effects of these substances on subsequent digestion and mass spectrometric analysis. However, the experimental conditions need to be altered for different protein samples. In particular, when the method is applied to the cleanup of SDS-solubilized membrane proteome samples, a series of problems exists, for example, how to precipitate/recover proteins and remove SDS efficiently, how to re-dissolve and digest the precipitated proteins with high efficiency, etc. In the study of Lin et al. (2012), the investigators optimized the experimental conditions for the application of acetone precipitation method to the cleanup of SDS-solubilized membrane protein samples, used enzyme- and MS-compatible SDC to overcome the problems of re-dissolution and digestion of acetone precipitated proteins, and thus developed an entirely solution-based combinative strategy that comprehensively utilizes the advantages of selected detergents and the optimized sample cleanup method to efficiently improve the analysis of membrane proteomes. This strategy overcomes some inherent limitations of the conventional sample preparation methods and is easily operated at low cost and suitable for the analysis of membrane proteomes varying in type and sample volume, etc.

\section{PVDF MEMBRANE-MEDIATED SAMPLE PREPARATION}

Because the majority of current proteomic strategies have limitations with regard to the direct analysis of intact proteins, proteolytic peptides obtained from various digestion procedures are frequently used for the identification of proteins in a typical proteomic analysis. In-gel enzymatic digestion of proteins following gel electrophoresis or gel-based sample cleanup is most commonly used in proteomic research. Although this method is straightforward and is suitable for the analysis of micro-amount samples, problems with incomplete protein digestion and low peptide recovery are common. These limitations of the method can be particularly problematic for the identification of high-molecular-weight or hydrophobic proteins, as well as for the characterization of posttranslational modifications (PTM) of proteins, because some important modified residues and peptides may be lost in the analytical procedure.

To overcome the limitations of in-gel digestion, some alternative methods, besides in-solution digestion, have been developed. These include transfer of the gel-separated intact proteins by electroblotting onto membranes such as nitrocellulose (NC) (Luque-Garcia et al., 2006, 2008) and polyvinyl difluoride (PVDF) (Bienvenut et al., 1999; Jonsson et al., 2001; Bunai et al., 2003), followed by proteolysis of the transferred proteins. PVDF membrane is used more frequently used than NC membrane because of the advantages of higher mechanical strength and binding force. After electroblotting onto a PVDF membrane, gel-separated proteins are generally extracted from the membrane using solutions containing detergents or organic solvents and then digested in-solution (Jonsson et al., 2001), or directly on-membrane, after which they are extracted (Bienvenut et al., 1999; Bunai et al., 2003). For example, in the experiment of Jonsson et al. (2001), the proteins electroblotted from gel onto a PVDF membrane were first extracted from it by incubation at $42^{\circ} \mathrm{C}$ overnight using a solution of $1 \%$ trifluoroacetic acid in $70 \%$ acetonitrile, and then digested in-solution, after which the extracts were dried and re-dissolved with $0.2 \mathrm{~mol} / \mathrm{L} \mathrm{NH}_{4} \mathrm{HCO}_{3}$. Although this method was reported to increase the protein coverage by an average of $30 \%$ compared with the conventional in-gel digestion approach, it has some drawbacks, such as being time-consuming and having a low efficiency of extraction of the proteins from the PVDF membrane, which affect the speed (high throughput) and sensitivity in identification of the proteins, especially those with high molecular weight and/or hydrophobicity. Bienvenut et al. (1999) used an on-membrane digestion method with $50 \mathrm{mmol} / \mathrm{L} \mathrm{NH}_{4} \mathrm{HCO}_{3}$ $30 \%$ acetonitrile (ACN) as a buffer solution in their experiments, and demonstrated that this method was similar to in-gel digestion in terms of sequence coverage and digestion efficiency of the tested proteins. Bunai et al. (2003) compared the on-membrane digestion efficiencies of PVDF-immobilized Bacillus subtilis SecA protein in the presence of $80 \%$ and $5 \%$ ACN. They concluded that the recovery of peptide fragments derived from the protein was higher in $80 \%$ than in $5 \% \mathrm{ACN}$. However, as with the in-gel digestion, the proteolytic enzyme in on-membrane digestion process does not have full access to the target proteins; as a result the large and/or hydrophobic peptides are difficult to extract from the membrane. Therefore, the digestion efficiency and peptide recovery are limited.

Lin et al. (2009) described a new dimethylformamide (DMF)-assisted digestion method for the mass spectrometric analysis of gel-separated proteins electroblotted onto a PVDF membrane. The method involved electroblotting gel-separa- 
ted proteins onto a PVDF membrane, excising the PVDF bands containing protein of interest, and dissolving the bands with pure DMF ( $\geqslant 99.8 \%$ ). Before tryptic digestion, $\mathrm{NH}_{4} \mathrm{HCO}_{3}$ buffer was added to moderately adjust the DMF concentration (to $40 \%$ ) in order for trypsin to exert its activity. Experimental results using standard proteins showed that, due to the ability of DMF to dissolve both PVDF membrane and the membrane-bound proteins, the proteins were digested virtually in-solution in DMF-containing buffers, which allowed more efficient protein digestion and peptide recovery, thereby increasing the sequence coverage and the confidence of protein identification.

\section{MILD PERFORMIC ACID OXIDATION-ASSISTED DIGESTION}

The solubilization of membrane proteins is a prerequisite to the efficient digestion of these proteins. Besides using additives such as detergents to improve the solubility of the membrane proteins, structural modification may also be helpful. Eichacker et al. (2004) showed that nearly $50 \%$ of all TMDs in the eukaryotic proteome contain a Met, and proposed that methionine modification (e.g., by oxidation or $\mathrm{CNBr}$ digestion) could be a tool to increase the number of TMDs by detected by MS. Performic acid oxidation, a method developed during the 1960s, completely oxidizes methionine to the sulfoxide and cysteine to cysteic acid (Hirs, 1967; Finlayson, 1969). Therefore, it can be used to shift the hydrophobicity of transmembrane peptides because of the hydrophilic nature of the oxidized peptides, thereby increasing accessibility for proteolytic attack (Eichacker et al., 2004). Unfortunately, the traditional performic acid oxidation can affect other amino acids, especially tryptophan, as well, and this restricts its application in proteomics. Pesavento et al. (2007) described and applied a mild performic acid oxidation method in a top-down proteomic study and found no side-reactions. In the report of Cao et al. (2010), a new mild performic acid oxidation-assisted digestion strategy was described that greatly improves the identification of a larger number of hydrophobic multi-spanning IMPs by oxidation of methionine. The method focuses on reducing the hydrophobicity of the peptides rather than the length of the transmembrane peptides. The experimental results showed that, by using the strategy, the hydrophobicity of IMPs was significantly decreased by oxidizing their methionine and cysteine residues with performic acid, thereby improving the solubility and proteolysis of these proteins. The application of the strategy to the analysis of IMPs from human nasopharyngeal carcinoma CNE1 cell line demonstrated that many IMPs, including those with high hydrophobicity, could be reliably identified.

\section{SUMMARY AND CONCLUSION}

The special properties, primarily the low abundance and high hydrophobicity of most membrane proteins, make their analysis more difficult than that of common soluble proteins. This mini-review is written to give some insight into membrane sample preparation strategies and focuses on the two main issues rather than covering all the advances in the membrane proteomics. To date, many strategies have been developed to enrich low-abundance membrane proteins at membrane and/or protein levels. When the proteins are enriched at the membrane level, density gradient centrifugation following differential centrifugation is commonly used. However, using these methods alone, it is usually difficult to prepare a particular membrane sample with very high purity, due to the similarities in physicochemical properties of membranes of various subcellular organelles. Other strategies such as two-phase partitioning and affinity purification are used in combination with the conventional approaches to further purify the samples. Furthermore, the affinity purification is also the primary method for the enrichment of membrane proteins at the protein level. The enrichment of plasma membranes has been highly developed than for other cellular inner membranes.

Repeated washing of the prepared membrane debris with high salt and/or high $\mathrm{pH}$ solutions, and on-membrane pre-digestion with proteases (membrane shaving) can be employed as the additional steps to enrich integral membrane protein preparations. At the same time, membrane shaving is one of the strategies for the identification of integral membrane proteins, because the soluble domains cleaved from the proteins by "shaving" can provide partial sequence information.

Membrane proteins can be digested in gel (in-gel digestion), in solution (in-solution digestion) after being extracted from the membranes, or on the membranes (on-membrane digestion) without extraction in advance. The membrane protein digestion should be carried out in the presence of additives that are compatible with enzyme activity, as common aqueous buffers cannot efficiently solubilize the hydrophobic membrane proteins. When extracting the membrane proteins, it is necessary to use agents that both can efficiently extract the membrane proteins and are compatible with the subsequent processes, including proteolysis and MS analysis, though the number of such agents is limited. Otherwise, the agents with strong ability to solubilize the membranes and extract membrane proteins, such as SDS, should be used, followed by sample cleanup. It is worth mentioning that when the 'cleaned' membrane proteins are digested, addition of some enzyme activity-compatible additives is helpful to the dissolution and proteolysis of these proteins. Many methods, including traditional and newly developed ones, have been employed to clean up protein samples. However, all the reported methods have inherent advantages and limitations. To date, no method has been described that is able to solve all the problems and more efforts are needed in the field of sample preparation for the analysis of membrane proteomes (Table 1). 
Table 1. Comments on the main agents/methods used for membrane protein sample preparation

\begin{tabular}{|c|c|}
\hline SDS (sodium dodecylsulfate) & $\begin{array}{l}\text { With the strongest ability to disrupt membrane and extract the proteins; severely reduces protease activity } \\
\text { and interferes with LC-MS analysis at } \geq 0.1 \% \text {, usually needed to be removed prior to enzymolysis and sub- } \\
\text { sequent analyses. }\end{array}$ \\
\hline SDC (sodium deoxycholate) & $\begin{array}{l}\text { Compatible with the activities of commonly used proteases up to } 2 \% \text {, removed by acidification, with the } \\
\text { membrane disruption ability being weaker than that of SDS. }\end{array}$ \\
\hline ALS (RapidGest SF) & $\begin{array}{l}\text { With moderate membrane disruption ability, compatible with the activities of commonly used proteases at } \\
\sim 0.1 \% \text {, degraded by acidification, commercial reagent specific for membrane proteomics, with a high price. }\end{array}$ \\
\hline Tube gel digestion & $\begin{array}{l}\text { Used for the cleanup of sample, apt to cause a certain sample loss and adverse protein modifications, difficult } \\
\text { to avoid the limitations of in-gel digestion. }\end{array}$ \\
\hline Dried gel absorption & $\begin{array}{l}\text { Used for the cleanup of sample, with ability to avoid sample loss and adverse protein modifications, operates } \\
\text { more convenient than tube gel digestion, difficult to avoid the limitations of in-gel digestion. }\end{array}$ \\
\hline Spin filter-based method & $\begin{array}{l}\text { Utilizes filter unit as a reactor for detergent removal, protein digestion and separation of digested peptides, } \\
\text { low peptide/protein recovery, not suitable for small amounts of samples. }\end{array}$ \\
\hline $\begin{array}{l}\text { Entirely solution-bas } \\
\text { combinative method }\end{array}$ & $\begin{array}{l}\text { Comprehensively utilizes the advantages of selected detergents and the optimized acetone precipitation, } \\
\text { operates easily and at low coat, particularly suitable for treating samples in large volume, apt to cause a } \\
\text { certain sample loss during acetone precipitation. }\end{array}$ \\
\hline
\end{tabular}

\section{ACKNOWLEDGEMENTS}

This work was supported by grants from Hunan Provincial Natural Science Foundation of China (No. 11JJ2019), National Basic Research Program of China (No. 2010CB529800), National Natural Science Foundation of China (Grant No. 31070700), and Specialized Research Foundation for the doctorial Program of Higher Education of China (No. 20094306110003).

\section{ABBREVIATIONS}

ACN, acetonitrile; DMF, dimethylformamide; GGE, gradient gel electrophoresis; HPLC, high-performance liquid chromatography; IMP, integral membrane protein; NC, nitrocellulose; $P E G$, polyethylene glycol; PM, plasma membrane; PVDF, polyvinyl difluoride; SDC, sodium deoxycholate; TMD, transmembrane domain

\section{REFERENCES}

Andersen, P., and Heron, I. (1993). Simultaneous electroelution of whole SDS-polyacrylamide gels for the direct cellular analysis of complex protein mixtures. J Immunol Methods 161, 29-39.

Bartee, E., McCormack, A., and Früh, K. (2006). Quantitative membrane proteomics reveals new cellular targets of viral immune modulators. PLoS Pathog 2, 975-988.

Bienvenut, W.V., Sanchez, J. C., Karmime, A., Rouge, V., Rose, K., Binz, P.A., and Hochstrasser, D.F. (1999). Toward a clinical molecular scanner for proteome research: parallel protein chemical processing before and during western blot. Anal Chem 71, 4800-4807.

Blonder, J., Goshe, M.B., Moore R.J., Pasa-Tolic, L., Masselon, C.D., Lipton, M.S., and Smith, R.D. (2002). Enrichment of integral membrane proteins for proteomic analysis using liquid chromatography-tandem mass spectrometry. J Proteome Res 1, 351-360.

Botelho, D., Wall, M.J., Vieira, D.B., Fitzsimmons, S., Liu, F., and Doucette, A. (2010). Top-down and bottom-up proteomics of SDS-containing solutions following mass-based separation. J Proteome Res 9, 2863-2870.

Bunai, K., Nozaki, M., Hamano, M., and Ogane, S. (2003). Proteomic analysis of acrylamide gel separated proteins immobilized on polyvinylidene difluoride membranes following proteolytic digestion in the presence of $80 \%$ acetonitrile. Proteomics 3, 1738-1749.

Cao, R., Liu, Y.S., Chen, P., Lv, R., Song, Q., Sheng, T.T., He, Q.Y., Wang, Y., Wang, X.C., and Liang, S.P. (2010). Improvement of hydrophobic integral membrane protein identification by mild performic acid oxidation-assisted digestion. Anal Biochem 407, 196-204.

Cao, R., Li, X., Liu, Z., Peng, X., Hu, W., Wang, X., Chen, P., Xie, J., and Liang, S. (2006). Integration of a two-phase partition method into proteomics research on rat liver plasma membrane proteins. $J$ Proteome Res 5, 634-642.

Eichacker, L.A., Granvogl, B., Mirus, O., Muller, B.C., Miess, C., and Schleiff, E. (2004). Hiding behind hydrophobicity: transmembrane segments in mass spectrometry. J Biol Chem 279, 50915-50922.

Finlayson, A.J. (1969). The performic acid oxidation of egg-white lysozyme. Can J Bio Chem 47, 31-37.

Fischer, F., Wolters, D., Rögner, M., and Poetsch, A. (2006). Toward the complete membrane proteome: high coverage of integral membrane proteins through transmembrane peptide detection. Mol Cell Proteomics 5, 444-453.

Hirs, C.H.W. (1967). Performic acid oxidation, Methods Enzymol 11, 197-199.

Hudgin, R.L., and Ashwell, G. (1974). Studies on the role of glycosyltransferases in the hepatic binding of asialoglycoproteins. J Biol Chem 249, 7269-7272.

Jonsson, A.P., Aissouni, Y., Palmberg, C., Percipalle, P., Percipalle, P., Nordling, E., Daneholt, B., Jornvall, H., and Bergman, T. (2001). Recovery of gel-separated proteins for in-solution digestion and mass spectrometry. Anal Chem 73, 5370-5377.

Liebler, D.C., and Ham, A.J. (2009). Spin filter-based sample preparation for shotgun proteomics. Nat Methods 6, 785.

Lin, Y., Liu, H., Liu, Z.H., Wang, X.C., and Liang, S.P. (2012). Shotgun analysis of membrane proteomes using a novel combinative strategy of solution-based sample preparation coupled with liquid chromatography-tandem mass spectrometry. J Chromatogr B Analyt Technol Biomed Life Sci 901, 18-24.

Lin, Y., Liu, Y., Li, J.J., Zhao, Y., He, Q.Z., Han, W.J., Chen, P., Wang, X.C., and Liang, S.P. (2010). Evaluation and optimization of removal of an acid-insoluble surfactant for shotgun analysis of 
membrane proteome. Electrophoresis 31, 2705-2713.

Lin, Y., Li, Y., Liu, Y., Han, W.J., He, Q.Z., Li, J.L., Chen, P., Wang, X.C., and Liang, S.P. (2009). Improvement of gel-separated protein identification by DMF-assisted digestion and peptide recovery after electroblotting. Electrophoresis 30, 3626-3635.

Lin, Y., Zhou, J., Bi, D., Chen, P., Wang , X.C., and Liang , S.P. (2008). Sodium deoxycholate-assisted tryptic digestion and identification of proteolytically resistant proteins. Anal Biochem 377 , 259-266.

Liu, T., Martin, A.M., Sinai, A.P., and Lynn, B.C. (2008). Three-layer sandwich gel electrophoresis: a method of salt removal and protein concentration in proteome analysis. J Proteome Res 7, $4256-4265$.

Liu,Y., Lin,Y., Yan,Y.Z., Li, J.L., He, Q.Z., Chen, P., Wang, X.C., and Liang, S.P. (2012). Electrophoretically-driven SDS removal and protein fractionation in the shotgun analysis of membrane proteomes. Electrophoresis 33, 316-324.

Li, X., Jia, X., Xie, C., Lin, Y., Cao, R., He, Q., Chen, P., Wang, X., and Liang, S. (2009). Development of cationic colloidal silica-coated magnetic nanospheres for highly selective and rapid enrichment of plasma membrane fractions for proteomics analysis. Biotechnol Appl Biochem 54, 213-320.

Luque-Garcia, J.L., Zhou, G., Spellman, D.S., Sun, T.T., and Neubert, T. A. (2008). Analysis of electroblotted proteins by mass spectrometry: protein identification after Western blotting. Mol Cell Proteomics 7, 308-314.

Luque-Garcia, J.L., Zhou, G., Sun,T.T., and Neubert, T.A. (2006). Use of nitrocellulose membranes for protein characterization by matrix-assisted laser desorption/ionization mass spectrometry. Anal Chem 78, 5102-5108.

Lu, X.N., and Zhu, H.N. (2005). Tube-gel digestion: a novel proteomic approach for high throughput analysis of membrane proteins. Mol Cell Proteomics 4, 1948-1958.

Manza, L.L., Stamer S.L., Ham A.J.L., Codreanu S.G., and Liebler, D.C. (2005) Sample preparation and digestion for proteomic analyses using spin filters. Proteomics 5, 1742-1745.

Marmagne, A., Rouet, M.A., Ferro, M., Rolland, N., Alconm, C., Joyard, J., Garin, J., Barbier-Brygoo, H., and Ephritikhine, G. (2004). Identification of new intrinsic proteins in Arabidopsis plasma membrane proteome. Mol Cell Proteomics 3, 675-691.

Masuda, T.,Tomita,M., and Ishihama,Y. (2008). Phase transfer surfactant-aided trypsin digestion for membrane proteome analysis. $\mathrm{J}$ Proteome Res 7, 731-740.

Morré, D.J., and Morré, D.M. (1989). Preparation of mammalian plasma membranes by aqueous two-phase partition. Biotechniques 7, 946-948.

Nielsen, P. A., Olsen, J.V., Podtelejnikov, A.V., Andersen, J.R., Mann, M., and Wiśniewski, J.R. (2005). Proteomic mapping of brain plasma membrane proteins. Mol Cell Proteomics 4, 402-408.

Pesavento, J.J., Garcia, B.A., Streeky, J.A., Kelleher, N.L., and Mizzen, C.A. (2007). Mild performic acid oxidation enhances chromatographic and top down mass spectrometric analyses of histones. Mol Cell Proteomics 6, 1510-1526.

Puchades, M., Westman, A., Blennow, K., and Davidsson,P. (1999). Removal of sodium dodecyl sulfate from protein samples prior to matrix-assisted laser desorption/ionization mass spectrometry. Rapid Commun. Mass Spectrom 13, 344-349.

Reinders, J., Zahedi, R.P., Pfanner, N., Meisinger, C., and Sickmann, A. (2006). Toward the complete yeast mitochondrial proteome: multidimensional separation techniques for mitochondrial proteomics. J Proteome Res. 5, 1543-1554.

Reynolds, J.A., and Tanford, C. (1970). The gross conformation of protein-sodium dodecyl sulfate complexes. J Biol Chem 245, 5161-5165.

Schindler, J., Lewandrowski, U., Sickmann, A., and Friauf, E. (2006). Proteomic analysis of brain plasma membrane isolated by affinity two-phase partitioning. Mol Cell Proteomics 5, 390-400.

Schindler, J., and Nothwang, H.G. (2006). Aqueous polymer two-phase systems: effective tools for plasma membrane proteomics. Proteomics 6, 5409-5417.

Speers, A.E., and Wu, C.C. (2007). Proteomics of integral membrane proteins-theory and application. Chem Rev 107, 3687-3714.

Walter, H., Walter, H., Brooks, D.E., and Fisher, D. (1985). Partitioning in aqueous two-phase systems: theory, methods, uses and applications to biotechnology. (Orlando, Academic Press), pp. 327.

Washburn, M.P., Wolters, D., and Yates III, J.R. (2001). Large-scale analysis of the yeast proteome by multidimensional protein identification technology.Nat Biotechnol. 19, 242-247.

Wiśniewski, J.R., Zougman, A., Nagaraj, N., and Mann, M. (2009). Universal sample preparation method for proteome analysis. Nat Methods 6, 359-362.

Wu, C.C., MacCoss, M.J., Howell, K.E., Yates, J.R. 3rd. (2003). A method for the comprehensive proteomic analysis of membrane proteins. Nat Biotechnol 21, 532-538.

Xiong, X., Huang, S., Zhang, H., Li, J.J., Shen, J.Y., Xiong, J.X., Lin, Y., Jiang, L.P., Wang, X.C., and Liang, S.P. (2009). Enrichment and proteomic analysis of plasma membrane from rat dorsal root ganglion neurons. Proteome Sci 7, 41-50.

Zhang, L., Wang, X., Peng, X., Wei, Y., Cao, R., Liu, Z., Xiong, J., Ying, X., Chen, P., and Liang S. (2007). Immunoaffinity purification of plasma membrane with secondary antibody superparamagnetic beads for proteomic analysis. J Proteome Res 6, 34-43.

Zhou, J., Li, J.L., Li, J.J., Chen, P., Wang, X.C., and Liang, S.P. (2010a). Dried polyacrylamide gel absorption: A method for efficient elimination of the interferences from SDS-solubilized protein samples in mass spectrometry-based proteome analysis. Electrophoresis 31, 3816-3822.

Zhou, J., Lin, Y., Deng, X.C., Shen, J.Y., He, Q.Y., Chen, P., Wang, X.C., and Liang, S.P. (2008). Development and application of a two-phase, on-membrane digestion method in the analysis of membrane proteome. J Proteome Res 7, 1778-1783.

Zhou, J., Xiong, J., Li, J., Huang, S., Zhang, H., He, Q., Lin, Y., Chen, P., Wang, X., and Liang, S. (2010b). Gel absorption-based sample preparation for the analysis of membrane proteome by mass spectrometry. Anal Biochem 404, 204-210.

Zhou, J., Zhou, T.Y., Cao, R., Liu, Z., Shen, J.Y., Chen, P., Wang, X.C., and Liang, S.P. (2006). Evaluation of the application of sodium deoxycholate to proteomic analysis of rat hippocaampal plasma membrane. J Proteome Res 5, 2547- 2553. 